07,05

\title{
Влияние механических напряжений и отжига на магнитную структуру и магнитоимпеданс аморфных CoFeSiBCr микропроводов
}

\author{
(C) М.Г. Неьматов 1,3 , М.М. Салем ${ }^{1}$, У. Азим ${ }^{1}$, М. Ахмат ${ }^{1}$, А.Т. Морченко ${ }^{1}$, \\ Н.А. Юданов ${ }^{1}$, Л.В. Панина ${ }^{1,2}$ \\ ${ }^{1}$ Национальный исследовательский технологический университет „МИСиС“, \\ Москва, Россия \\ ${ }^{2}$ Институт проблем проектирования в микроэлектронике РАН, \\ Москва, Россия \\ ${ }^{3}$ Таджикский технический университет им. акад. М.С. Осими, \\ Душанбе, Таджикистан \\ E-mail: nematovmaqsud@misis.ru, Ipanina@plymouth.ac.uk,
}

(Поступила в Редакцию 1 августа 2017 г.)

Структурные и магнитные свойства аморфных ферромагнитных микропроводов могут претерпевать значительные изменения в результате воздействия внешних механических напряжений и температурной обработки. Изучение происходящих при этом трансформаций представляется важным как для разработки различных сенсоров механических напряжений, нагрузки, температуры, так и для индуцирования в проводах определенного типа магнитной анизотропии, играющей значительную роль в осуществлении в них различных эффектов. В настоящей работе исследовано влияние внешних напряжений и отжига на процессы намагничивания и магнитный импеданс в микропроводах состава $\mathrm{Co}_{71} \mathrm{Fe}_{5} \mathrm{~B}_{11} \mathrm{Si}_{10} \mathrm{Cr}_{3}$, которые в аморфном состоянии имеют невысокую положительную магнитострикцию (порядка $10^{-8}$ ). Воздействие внешних напряжений приводит к резкому изменению характера кривой перемагничивания, что обусловлено изменением знака магнитострикции и типа магнитной анизотропии. Соответственно амплитуда высших гармоник и величина магнитного импеданса оказываются чувствительными к механическим напряжениям. В проводах с частичной кристаллизацией действие упругих напряжений не приводит к заметному изменению магнитных свойств, однако с помощью отжига можно добиться существенного увеличения осевой магнитной анизотропии проводов, находящихся в напряженном состоянии. Экспериментальные результаты проанализированы в рамках магнитострикционной модели наведенной магнитной анизотропии.

DOI: 10.21883/FTT.2018.02.45387.234

\section{1. Введение}

Ферромагнитные микропровода с аморфной или нанокристаллической структурой имеют ряд специфических магнитных свойств: наличие двух устойчивых состояний намагниченности (бистабильность), высокую подвижность доменов и гигантский магнитный импеданс (МИ) [1-6].

Явление магнитной бистабильности, сопровождающееся быстрым распространением одиночных доменов вдоль оси провода, наблюдается в микропроводах из сплавов с высоким содержанием железа. Для них характерна положительная магнитострикция, которая в сочетании с растягивающими напряжениями, образующимися в процессе быстрого затвердевания при вытягивании из расплава, приводит к осевой магнитной анизотропии. При перемагничивании таких проводов в продольном направлении наблюдается почти прямоугольная петля гистерезиса и генерируется узкий сигнал электрического напряжения, имеющий в спектре гармоники высших порядков. Так как на высоких частотах подавляется обычный шум и $1 / f$ шум, то появляется возможность детектировать такие гармоники с высокой точностью, что предложено использовать в различных сенсорных системах [7-9].
Другой тип аморфных микропроводов, приготовленных из сплавов на основе кобальта, имеет отрицательную магнитострикцию и циркулярную магнитную анизотропию. В результате при перемагничивании в осевом магнитном поле наблюдается наклонная кривая намагничивания с незначительным гистерезисом и высокой магнитной восприимчивостью. В этих материалах проявляется МИ эффект - очень большое изменение высокочастотного импеданса микропровода под влиянием продольного магнитного поля. Указанное свойство используется при разработке высокочувствительных магнитных датчиков для регистрации магнитных полей чрезвычайно низкой напряженности (см., например, обзор [10]).

При отсутствии магнитокристаллической анизотропии в аморфном состоянии основной вклад в формирование магнитной структуры вносят магнитострикционные взаимодействия. Это приводит к зависимости упомянутых выше эффектов от внешних механических напряжений, что используют для создания сенсоров различных механических величин $[11,12]$. Кроме того, магнитные и структурные свойства проводов можно регулировать с помощью специальных тепловых обработок, в результате которых происходит релаксация внутренних напряжений или индуцирование таковых в определенном направлении, а в некоторых случаях и частичная 
кристаллизация изначально аморфного сплава [13-16]. Как правило, усилия исследователей направлены на достижение определенного максимального эффекта путем совершенствования магнитной структуры провода. Например, в работе [14] у микропроводов на основе Со с отрицательной магнитострикцией в результате отжига электрическим током достигается циркулярная магнитная анизотропия с малым разбросом осей анизотропии и небольшой константой анизотропии, что привело к рекордным изменениям МИ (около 600\% на частоте $15 \mathrm{MHz}$ ).

В данной работе исследуется влияние внешних механических напряжений и отжига на магнитную структуру микропроводов на основе железо-кобальтовых сплавов, имеющих небольшую положительную магнитострикцию и осевую магнитную анизотропию. Под действием внешних напряжений константа магнитострикции изменяет знак, что сопровождается скачкообразным изменением типа анизотропии, кривой намагничивания и МИ. Это приводит к усилению зависимости амплитуд гармонического спектра и МИ от механических напряжений. Отжиг стабилизирует осевую магнитную структуру, однако в проводах с частичной кристаллизацией после отжига наблюдается сильная зависимость коэрцитивной силы от внешних напряжений и возникает асимметрия кривой намагничивания. Обнаруженные явления могут быть перспективными для разработки миниатюрных сенсоров механических напряжений.

\section{2. Анализ статической намагниченности}

Для анализа магнитной структуры в аморфных проводах с учетом различных напряжений может быть предложена следующая модель. Предполагается, что в проводе имеются осевые напряжения растяжения или сжатия $\sigma_{a}=\sigma_{i}+\sigma_{e x}$, где $\sigma_{i}-$ внутренние напряжения, возникающие в процессе быстрого затвердевания аморфного сплава, $\sigma_{e x}$ - внешние растягивающие напряжения. Также предполагается наличие внутренних скручивающих напряжений, которые соответствуют воздействию растяжения и сжатия $\pm \sigma_{t}$, направленных перпендикулярно друг другу и под углом в $45^{\circ}$ по отношению к оси провода. Именно наличие скручивающих растяжений позволяет ввести понятие обобщенной геликоидальной магнитной анизотропии и описать с его помощью возможный переход от осевой к циркулярной анизотропии под действием внешних растяжений. Магнитная энергия $U_{m}$ записывается в виде

$$
\begin{aligned}
U_{m}= & -K \cos ^{2} \theta-\frac{3}{2} \lambda \sigma_{a} \cos ^{2} \theta \\
& -\frac{3}{2} \lambda \sigma_{t}\left[\cos ^{2}\left(\theta-\frac{\pi}{4}\right)-\cos ^{2}\left(\theta+\frac{\pi}{4}\right)\right] .
\end{aligned}
$$

Здесь $K-$ константа одноосной анизотропии, которая не связана с магнитострикционными взаимодействиями, $\theta$ - угол между намагниченностью и осью провода, $\lambda$ - константа магнитострикции, которая для аморфных сплавов может существенно зависеть от растягивающих напряжений [17-19]

$$
\lambda\left(\sigma_{e x}\right)=\lambda(0)-\beta \sigma_{e x} .
$$

Параметр $\beta$ находится в пределах $(1-6) \cdot 10^{-10} \mathrm{MPa}^{-1}$. Аналогичные эффекты зависимости магнитострикции от механических напряжений были обнаружены в нанокристаллических сплавах [20].

После несложных преобразований, выражение (1) можно записать в виде, характерном для одноосной анизотропии

$$
U_{m}=-|\tilde{K}| \cos ^{2}(\alpha-\theta),
$$

где $\alpha$ - угол наклона легкой оси анизотропии к оси провода, $\tilde{K}$ - эквивалентная константа одноосной анизотропии

$$
\tilde{K}=\frac{K+(3 / 2) \lambda \sigma_{a}}{\cos (2 \tilde{\alpha})}, \quad \tilde{\alpha}=\frac{1}{2} \operatorname{arctg} \frac{3\left|\lambda \sigma_{t}\right|}{\left|K+(3 / 2) \lambda \sigma_{a}\right|} .
$$

Угол $\alpha$ в уравнении (3) определяется следующим образом

$$
\begin{aligned}
& \text { a) } K+(3 / 2) \lambda \sigma_{a}>0, \alpha=\tilde{\alpha}, \\
& \text { b) } K+(3 / 2) \lambda \sigma_{a}=0, \alpha=45^{\circ}, \tilde{K}=3 \lambda \sigma_{t}, \\
& \text { c) } K+(3 / 2) \lambda \sigma_{a}<0, \alpha=90^{\circ}-\tilde{\alpha} .
\end{aligned}
$$

При положительных значениях констант магнитной анизотропии $K$ и магнитострикции $\lambda$ угол $\alpha<45^{\circ}$, и легкая ось анизотропии близка к оси провода, если $\sigma_{i}>\sigma_{t}$. При изменении знака магнитострикции под действием $\sigma_{e x}$ и выполнении условия $K-(3 / 2) \lambda \sigma_{a}>0$ легкая ось анизотропии приближается к циркулярному направлению. Одновременно с этим меняется характер кривых намагничивания: прямоугольная петля преобразуется в наклонную, область гистерезиса значительно уменьшается.

\section{3. Материалы и методики измерений}

Исследовались микропровода в стеклянной оболочке [21] состава $\mathrm{Co}_{71} \mathrm{Fe}_{5} \mathrm{~B}_{11} \mathrm{Si}_{10} \mathrm{Cr}_{3}$ с различной геометрией: общим диаметром 29.5 и $41.5 \mu \mathrm{m}$ и толщиной металлического сердечника 23.9 и $36.3 \mu \mathrm{m}$, обозначаемые в дальнейшем как образцы № 1 и 2 соответственно. Для сплава выбранного состава в аморфном состоянии характерна почти нулевая магнитострикция, порядка $10^{-8}-10^{-7}[22,23]$. При исследовании влияния механических напряжений к проводам прикладывались нагрузки до $80 \mathrm{~g}$ (что соответствует механическому напряжению в более тонком образце $1 \mathrm{GPa})$. Для модификации магнитной структуры провода отжигались при температуре $573 \mathrm{~K}$ в течение $60 \mathrm{~min}$. 

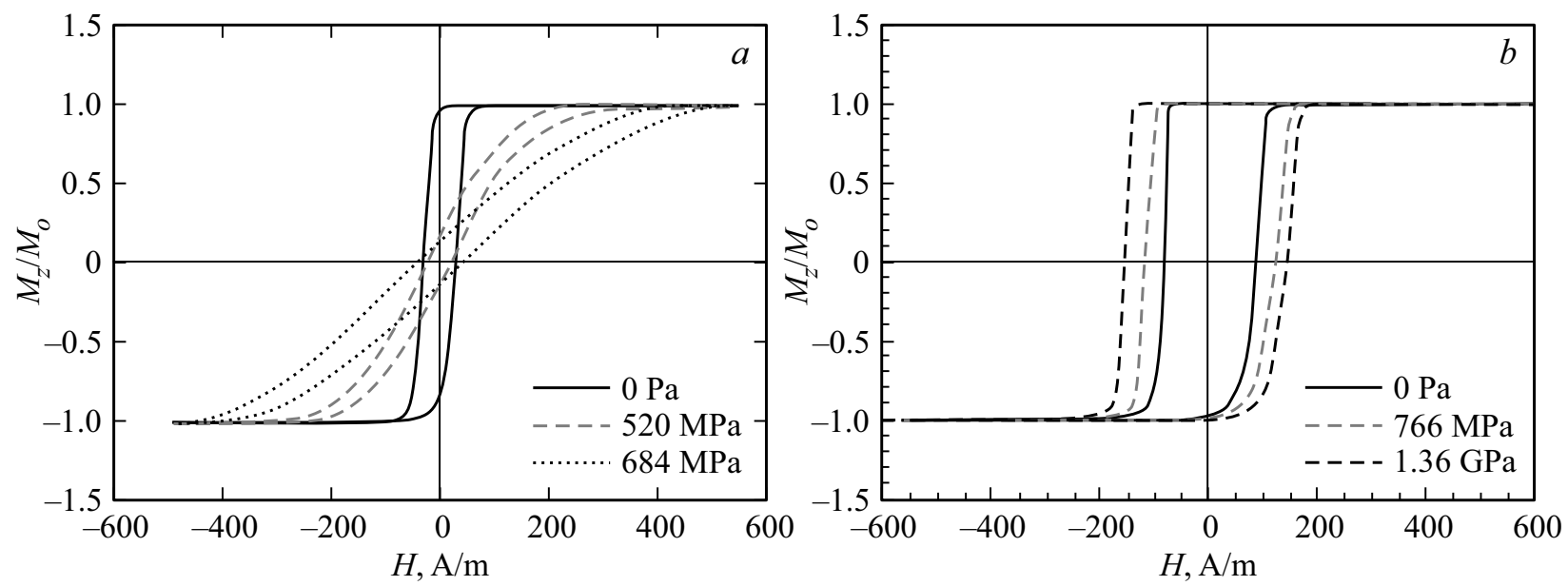

Рис. 1. Влияние растягивающего напряжения на петлю гистерезиса аморфного микропровода $\mathrm{Co}_{71} \mathrm{Fe}_{5} \mathrm{~B}_{11} \mathrm{Si}_{10} \mathrm{Cr}_{3}$ в стеклянной оболочке (образец № 1) при осевом намагничивании: $a$ ) в исходном состоянии; $b$ ) после отжига при $573 \mathrm{~K}$ в течение 60 min.
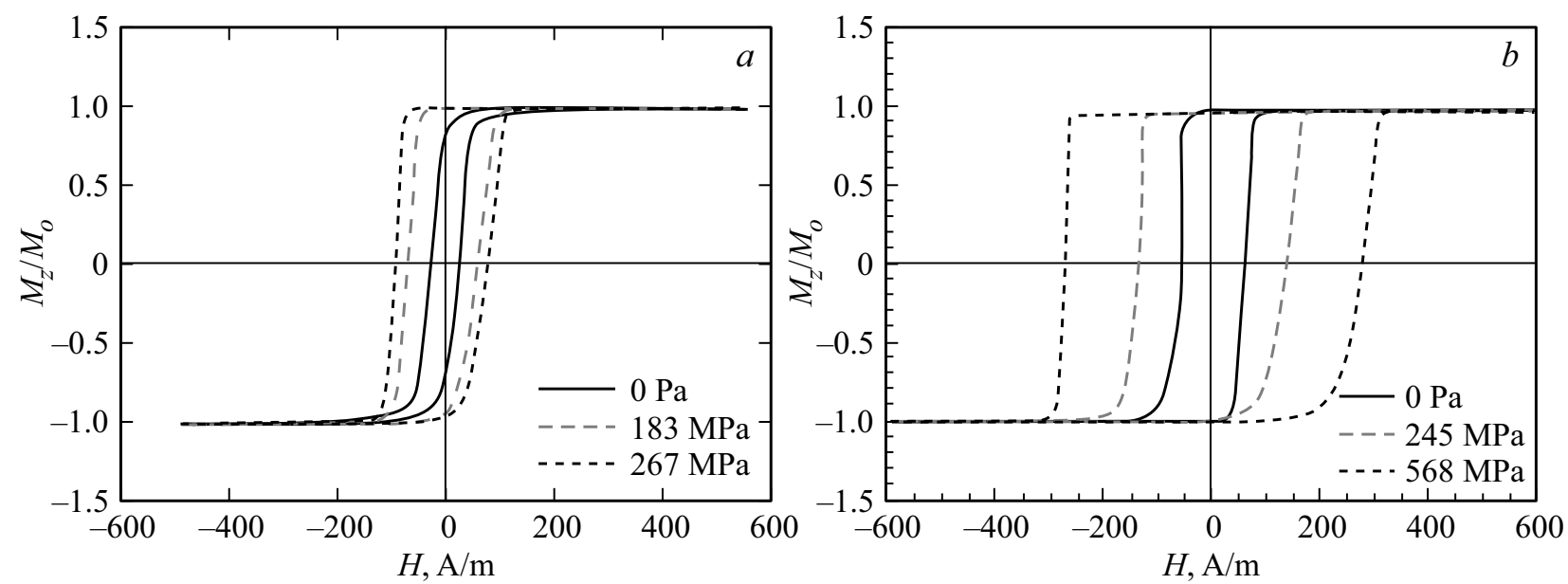

Рис. 2. Влияние растягивающего напряжения на петлю гистерезиса микропровода $\mathrm{Co}_{71} \mathrm{Fe}_{5} \mathrm{~B}_{11} \mathrm{Si}_{10} \mathrm{Cr}_{3}$ в стеклянной оболочке (образец № 2) при осевом намагничивании: $a$ ) в исходном состоянии, $b$ ) после отжига при $573 \mathrm{~K}$ в течение 60 тіn (частичная кристаллизация).

Структурные параметры проводов исследовались методом дифференциальной сканирующей калориметрии (ДСК). Измерения проводились в атмосфере Ar при скорости нагрева $10 \mathrm{~K} / \mathrm{min}$ с помощью высокочувствительного калориметра Netzsch DSC 204 F1 Phoenix. Температуры Кюри $\left(T_{c}=637 \mathrm{~K}\right)$ и кристаллизации $\left(T_{c r}=787 \mathrm{~K}\right)$ проводов были определены по графику ДСК с помощью стандартных приложений.

Процессы намагничивания проводов исследовались индукционным методом с использованием двух миниатюрных дифференциальных катушек с внутренним диаметром $3 \mathrm{~mm}$. Частота тока в намагничивающих катушках составляла $500 \mathrm{~Hz}$, максимальная амплитуда намагничивающего поля - $1000 \mathrm{~A} / \mathrm{m}$. Для построения петли гистерезиса индуцированный электрический сигнал оцифровывался и интегрировался как функция магнитного поля. Высшие гармоники в индуцированном при перемагничивании сигнале напряжения детектировались с помощью селективного усилителя (Signal Recovery 5210) и функциональных генераторов. Зависимость высокочастотного импеданса от магнитного поля исследовалась в диапазоне частот $1-100 \mathrm{MHz}$ с помощью векторного анализатора цепей (Hewlett-Packard 8753E) путем измерения параметра S12 (коэффициента передачи) в цепи, содержащей микропровод в специально разработанной микроволновой полосковой ячейке.

\section{4. Результаты и обсуждение}

Исследование кривых ДСК показывает, что оба типа проводов изначально обладали в основном аморфной структурой, а их кристаллизация проходит в два этапа. При этом на графиках для образца большего диаметра (№ 2) в области кристаллизации наблюдаются два широких пика, что свидетельствует о его частичной 
(около 15\%) кристаллизации в исходном состоянии. Это приводит к различным значениям константы магнитострикции: $1.2 \cdot 10^{-8}$ и $1.7 \cdot 10^{-6}$ в образцах № 1 и 2 соответственно. В исходном состоянии и в отсутствие внешних механических напряжений оба образца имеют почти прямоугольную петлю гистерезиса (рис. 1,a и 2,a) с небольшой коэрцитивностью (около $25 \mathrm{~A} / \mathrm{m}$ ), что обусловлено осевой анизотропией, характерной для $\lambda>0$. Под воздействием внешних механических напряжений кривая намагничивания для образца № 1 резко меняется и становится наклонной, если $\sigma_{e x}>200 \mathrm{MPa}$. Это можно объяснить изменением знака константы магнитострикции и переходом магнитной анизотропии от осевого типа к циркулярному, как следует из уравнений (4) и (5). С другой стороны, магнитные свойства образца № 2 под действием внешних механических напряжений не претерпевают существенных изменений, так как в нем характер магнитной анизотропии не изменяется.

Отжиг при температуре $T_{a n}=573 \mathrm{~K}$, значительно меньшей, чем температура кристаллизации, приводит к стабилизации осевой анизотропии и усилению бистабильных свойств, как видно из рис. $1, b$ и $2, b$. Можно предположить, что частичная релаксация внутренних
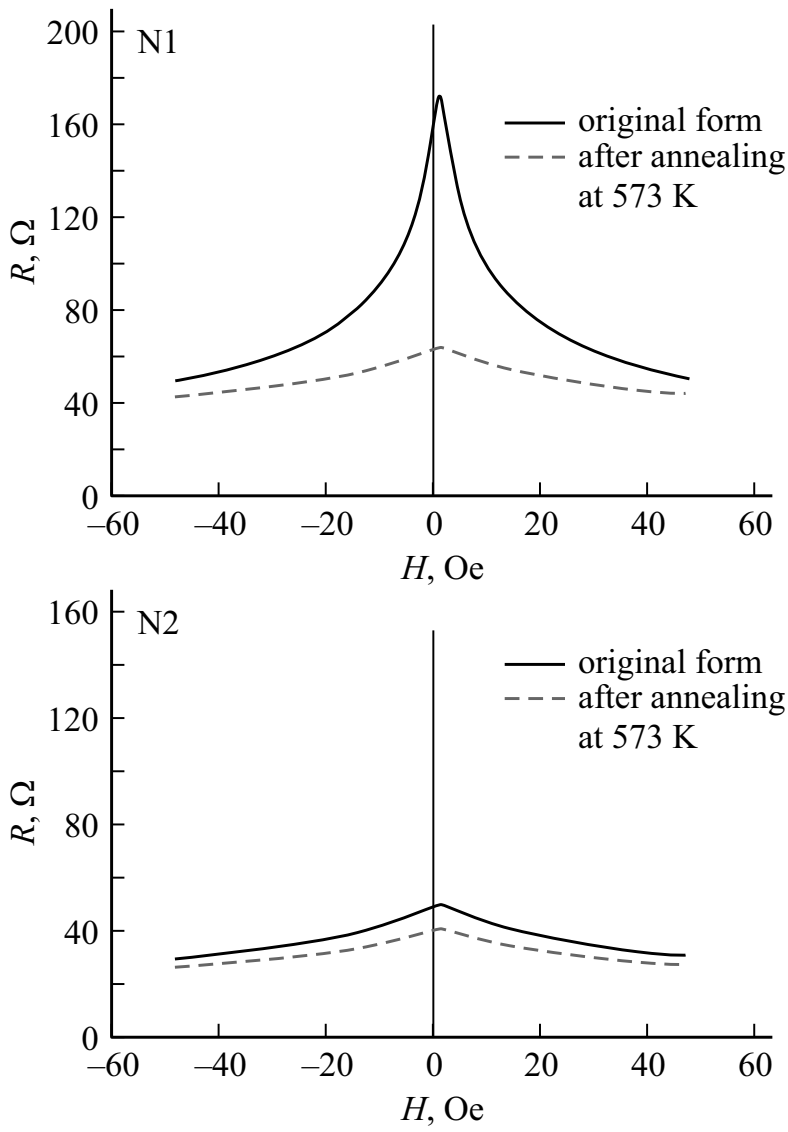

Рис. 3. Зависимость действительной части импеданса микропроводов $\mathrm{Co}_{71} \mathrm{Fe}_{5} \mathrm{~B}_{11} \mathrm{Si}_{10} \mathrm{Cr}_{3}$ (аморфного № 1 и частично кристаллизованного в результате отжига № 2) от магнитного поля в исходном состоянии и после отжига при $573 \mathrm{~K}$ в течение $60 \mathrm{~min}$. Частота тока возбуждения $50 \mathrm{MHz}$. напряжений при отжиге приводит к увеличению константы магнитострикции. Об этом также свидетельствует поведение кривых гистерезиса под нагрузкой для образца № 1, которые характеризуются бистабильностью и отличаются только небольшим увеличением коэрцитивной силы, т.е. в таких проводах изменения типа анизотропии не происходит. Интересно отметить, что для образца № 2 после отжига наблюдается весьма чувствительное поведение коэрцитивности по отношению к $\sigma_{e x}$. Видно также, что при увеличении $\sigma_{e x}$ кривые намагничивания становятся асимметричными. В этом случае сказывается влияние частичной кристаллизации, степень которой могла возрасти при отжиге, и различие поведения кристаллических и аморфных областей при их намагничивании [24].

Интересно сравнить поведение МИ в обоих типах образцов до и после отжига, так как это дает более полное представление о характере изменения анизотропии. Зависимости МИ от магнитного поля представлены на рис. 3. Для обоих типов проводов импеданс имеет максимум в нулевом поле, что характерно для систем с осью анизотропии, параллельной высокочастотному току и внешнему магнитному полю [6]. Значение импеданса в максимуме определяется магнитной восприимчивостью, которая на невысоких частотах (порядка десятков $\mathrm{MHz}$ ) увеличивается с уменьшением константы анизотропии. Как видно из рис. 3 , после отжига значение импеданса уменьшается приблизительно в 3 раза для образца № 1 и совсем незначительно изменяется для образца № 2. Таким образом, в первом случае происходит столь значительное усиление одноосной анизотропии, что внешние напряжения не могут оказать существенного влияния на поведение намагниченности.

\section{5. Ферромагнитные микропровода как сенсоры механических напряжений}

При перемагничивании бистабильных микропроводов генерируется узкий сигнал напряжения, имеющий в спектре гармоники высоких порядков. Амплитуда гармоник может зависеть от внешних параметров, таких как деформация, механическое напряжение, температура. Следовательно, подобные провода, установленные на поверхности изделий либо размещенные внутри материалов, могут использоваться в качестве датчиков с дистанционным опросом. Можно было бы предположить, что при наличии зависимости коэрцитивности от внешних факторов подобное поведение должны проявлять также и амплитуды высших гармоник. Однако, как показывает опыт, гармонический спектр слабо зависит от величины коэрцитивности и определяется типом кривых квазистатического намагничивания.

Как видно из рис. 4, $а$ наибольшие изменения в гармоническом спектре под действием механических напряжений наблюдаются для образца № 1 в исходном состоянии. Так, под действием нагрузки в $485 \mathrm{MPa}$ амплитуда 

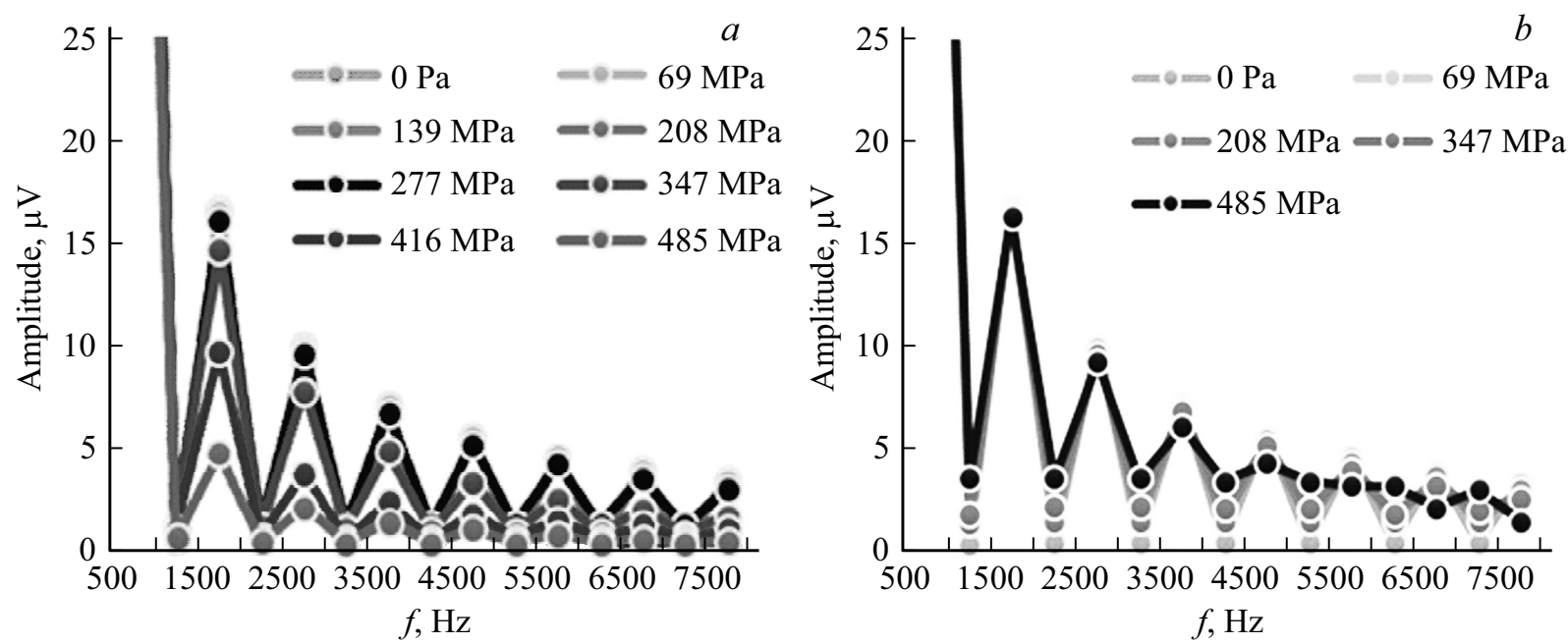

Рис. 4. Зависимость амплитуды высших гармоник от приложенного растягивающего напряжения для аморфного микропровода (образец № 1): $a$ ) в исходном состоянии, $b$ ) после отжига при $573 \mathrm{~K}$ в течение $60 \mathrm{~min}$.

3-й гармоники изменяется в 3 раза, а амплитуда 5-й гармоники - в 4 раза. Такое поведение обусловлено изменением типа кривых намагничивания при изменении знака магнитострикции. После отжига кривые намагничивания слабо изменяются при воздействии нагрузки, а соответственно слабо меняется и гармонический спектр. Это подтверждает вывод о том, что изменение гармонического спектра проводов в аморфном состоянии обусловлено именно изменением магнитострикции под действием механических напряжений.

Интересно отметить, что после отжига в спектре появляются четные гармоники, что обусловлено асимметрией кривых намагничивания (рис. $4, b$ ). Их амплитуды пропорциональны внешним механическим напряжениям. Наиболее значительно этот эффект выражен в частично кристаллизованном микропроводе (образец № 2 после отжига), процессы перемагничивания которого обнаруживают сильную асимметрию при увеличении $\sigma_{e x}$.

Изменение знака магнитострикции под действием нагрузки усиливает зависимость микроволнового импеданса от $\sigma_{e x}$, что также может быть использовано в сенсорных приложениях. Провод конечной длины ведет себя как симметричная антенна, параметр релаксации которой зависит от внешних факторов из-за модуляции импеданса. При переходе частоты возбуждения в микроволновую область основной вклад в МИ вносит изменение статической намагниченности под действием внешних факторов. Как видно из рис. 1, $a$, зависимость магнитострикции от внешней нагрузки приводит к соответствующей зависимости ориентации намагниченности, что и обеспечивает чувствительность МИ к изменению $\sigma_{e x}$.

\section{6. Заключение}

В работе было изучено влияние внешних механических напряжений и отжига на процессы намагничивания и магнитоимпеданс в аморфных и частично кристаллизованных микропроводах состава $\mathrm{Co}_{71} \mathrm{Fe}_{5} \mathrm{~B}_{11} \mathrm{Si}_{10} \mathrm{Cr}_{3}$, имеющих положительную магнитострикцию. Показано, что при увеличении нагрузки магнитострикция проводов в аморфном состоянии изменяет знак, что приводит к резкому изменению типа магнитной анизотропии и поведения кривых намагничивания. Это обусловливает высокую чувствительность гармонического спектра индуцированного сигнала напряжения и магнитоимпеданса к внешней нагрузке. Влияние отжига при температуpax, меньших температуры кристаллизации, усиливает осевую анизотропию, и при любых допустимых внешних механических напряжениях кривые намагничивания имеют бистабильный характер. Однако при увеличении нагрузки кривые намагничивания становятся асимметричными, а в гармоническом спектре появляются четные гармоники, амплитуды которых пропорциональны приложенным напряжениям. Обнаруженные эффекты могут быть перспективными для разработки миниатюрных встраиваемых сенсоров механических напряжений.

\section{Список литературы}

[1] M. Vazquez, H. Chiriac, A. Zhukov, L. Panina, T. Uchiyama. Phys. Status Solidi A 208, 493 (2011).

[2] A. Zhukov, V. Zhukova. Magnetic properties and applications of ferromagnetic microwires with amorpheous and nanocrystalline structure. Nova Science Publishers, N.Y. (2009).

[3] R. Varga, K.L. Garcia, M. Vazques. Phys. Rev. Lett. 94, 017201 (2005).

[4] M. Ipatov, N.A. Usov, A. Zhukov, J. González. Physica B 403, 379 (2008).

[5] L. Panina, K. Mohri. Appl. Phys. Lett. 65, 1189 (1994).

[6] D.P. Makhnovskiy, L.V. Panina, D.J. Mapps. Phys. Rev. B 63, 144424 (2001).

[7] K.G. Ong, M. Paulose, C.A. Grimes. Sensors 3, 11 (2003). 
[8] D. Markhnovskiy, N. Fry, A. Zhukov. Sensor Actuators A 166, 133 (2011).

[9] S.A. Baranov. Surf. Eng. Appl. Electrochem. 49, 189 (2013).

[10] K. Mohri, Y. Honkura, L.V. Panina, T. Uchiyama. J. Nanosci. Nanotechnology 12, 7491 (2012).

[11] K. Mohri, T. Uchiyama, L.P. Shen, C.M. Cai, L.V. Panina. Sensors Actuators 91, 1-2, 85 (2001).

[12] N. Bayri, S. Atalay. J. Alloy Comp. 381, 1-2, 245 (2004).

[13] C. Appino, C. Beatrice, P. Tiberto, F. Vinai. J. Magn. Magn. Mater. 215-216, 6, 349 (2000).

[14] L. Kraus, Z. Frait, K.R. Pirota, H. Chiriac. J. Magn. Magn. Mater. 254-255, 1, 399 (2003).

[15] J. Liu, F. Qin, D. Chen, H. Shen, H. Wang, D. Xing, M.-H. Phan. J. Sun. J. Appl. Phys. 115, 17, 17A326 (2014).

[16] V. Zhukova, A. Talaat, M. Ipatov, A. Zhukov. IEEE Transactions Magn. 50, 1 (2014).

[17] H. Szymczak. J. Magn. Magn. Mater. 67, 2, 227 (1987).

[18] J.M. Barandiarán, A. Hernando, V. Madurga, O.V. Nielsen, M. Vazguez, H. Vazguez-Lopez. Phys. Rev. B 35, 5066 (1987).

[19] A. Zhukov, M. Churyukanova, S. Kaloshkin, V. Sudarchikova, S. Gudoshnikov, M. Ipatov, A. Talaat, J.H. Blanco, V. Zhukova. J. Electronic Mater. 45, 1, 226 (2016).

[20] G. Herzer, S. Flohrer, C. Polak. IEEE Trans. Magn. 46, 2, 341 (2010).

[21] H. Chiriac, T.A. Ovari. Prog. Mater. Sci. 40, 333 (1996).

[22] M.M. Salem, M.G. Nematov, A. Uddin, L.V. Panina, M.N. Churyukanova, A.T. Morchenko. J. Phys. Conf. Ser. (2017). In press.

[23] М.М. Салем, М.Г. Неъматов, А. Уддин, Л.В. Панина, А.Т. Морченко, В.А. Скиданов. МЭС-2016. VII Всерос. научно-техн. конф. Сб. тр. 4, 207 (2016).

[24] N.A. Buznikov, C.G. Kim, C.O. Kim, S.S. Yoon. Phys. Met. Metallography 99, 1, S69 (2005). 